\title{
Morphology of human palmaris longus tendon
}

\author{
S. P. NICHOLLS, L. J. GATHERCOLE, AND J. S. SHAH \\ From the H. H. Wills Physics Laboratory, University of Bristol
}

SUMMARY A systematic morphological investigation of human palmaris longus tendons by polarisation microscopy and low angle $x$-ray diffraction is reported. It is shown that contrary to some previously reported observations, and in common with other tension bearing soft collagenous tissues, the fibres in this tendon are crimped. A new method of preparation of the tissue enabling one to see directly the crimped organisation in scanning electron microscopy was used to reinforce the findings by other methods.

Tendons and ligaments consist largely of collagen fibres organised in an essentially parallel array along the long axis of the tendon. These fibres, however, show undulations along their length which have periodicities of 10-100 $\mu \mathrm{m}$ depending on the type of tissues and species. According to the earlier writers ${ }^{12}$ the undulations in the tendons and other tissues were thought to be due to helical twisting of the constituent fibres.

Later morphological investigations ${ }^{3-7}$ have, however, established that the undulations in the fibres of a large number of tissues in different species (including humans) are not helical in nature but represent features called crimps, which are zigzag ribbon-like waveforms with crimp-like apices (Fig. 1). The crimps are shown to be of considerable importance in determining the mechanical properties of tendons, especially the nonlinear portion of the stress-strain curve. ${ }^{6-8}$

The evidence for the essential features of the crimped waveform, namely, (1) crimped apices, (2) nonhelical and planar nature, is considerable. In polarisation microscopy (PM) crimps are visualised as periodic light and dark bands (Fig. 2) along the fibre axes. The systematic variations of these bands caused by the rotation of the fibre along the axis of the polarising microscope show that the apices of the crimps are not like those in smooth undulations of a sine wave..$^{349}$ The conclusion derived from PM that the crimps are planar has been confirmed by lowangle $x$-ray diffraction ${ }^{5}$ and transmission electron microscopy..$^{11}{ }^{11}$ Evans and Barbanel ${ }^{12}$ have also confirmed the presence of the planar features of the crimps and the absence of helical waveforms in rat

Accepted for publication 19 August 1983.

Correspondence to Dr J. S. Shah, H. H. Wills Physics Laboratory, Royal Fort, Tyndall Avenue, Bristol BS8 1TL.

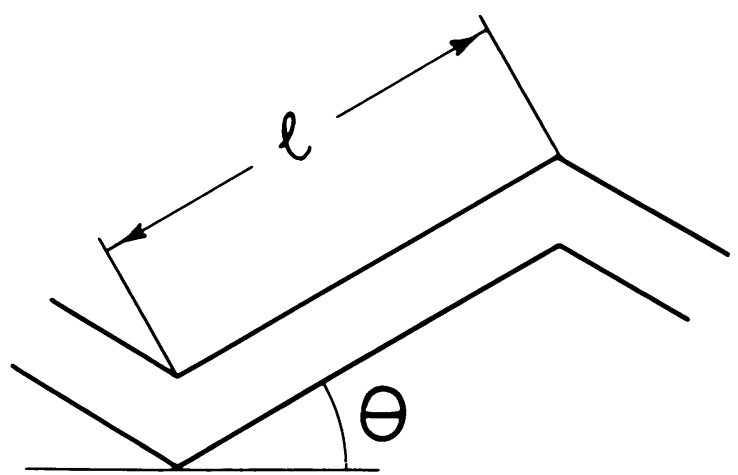

Fig. 1 Schematic diagram of a crimp showing crimp angle $\theta$ and crimp segment length $l$.

tail tendon (RTT) by scanning electron microscopy (SEM) of the silicone elastomer replicas of fresh, unfixed, RTT. Gathercole et al. ${ }^{13}$ have shown conclusive correlation of crimped waveforms, as visualised by SEM, of teased and freeze-dried fibres RTT with the light and dark bands seen in PM. Finally Shah et $a l .{ }^{7}$ have shown that the crimps are present in human anterior longitudinal ligaments in their natural wethydrated state by using a recently developed technique called moist environment ambient temperature scanning electron microscopy. ${ }^{14}{ }^{15}$

It has been suggested, ${ }^{12}$ however, that crimps are not present in the fibres of human palmaris longus tendons. The main evidence for this conclusion was SEM studies of hyaluronidase treated tissues. Hyaluronidase treatment of the tissues was necessary as direct visualisation of the morphology of fibres by SEM in intact and undamaged tissues is seldom possible, since these tissues have a fibrous sheath or a 

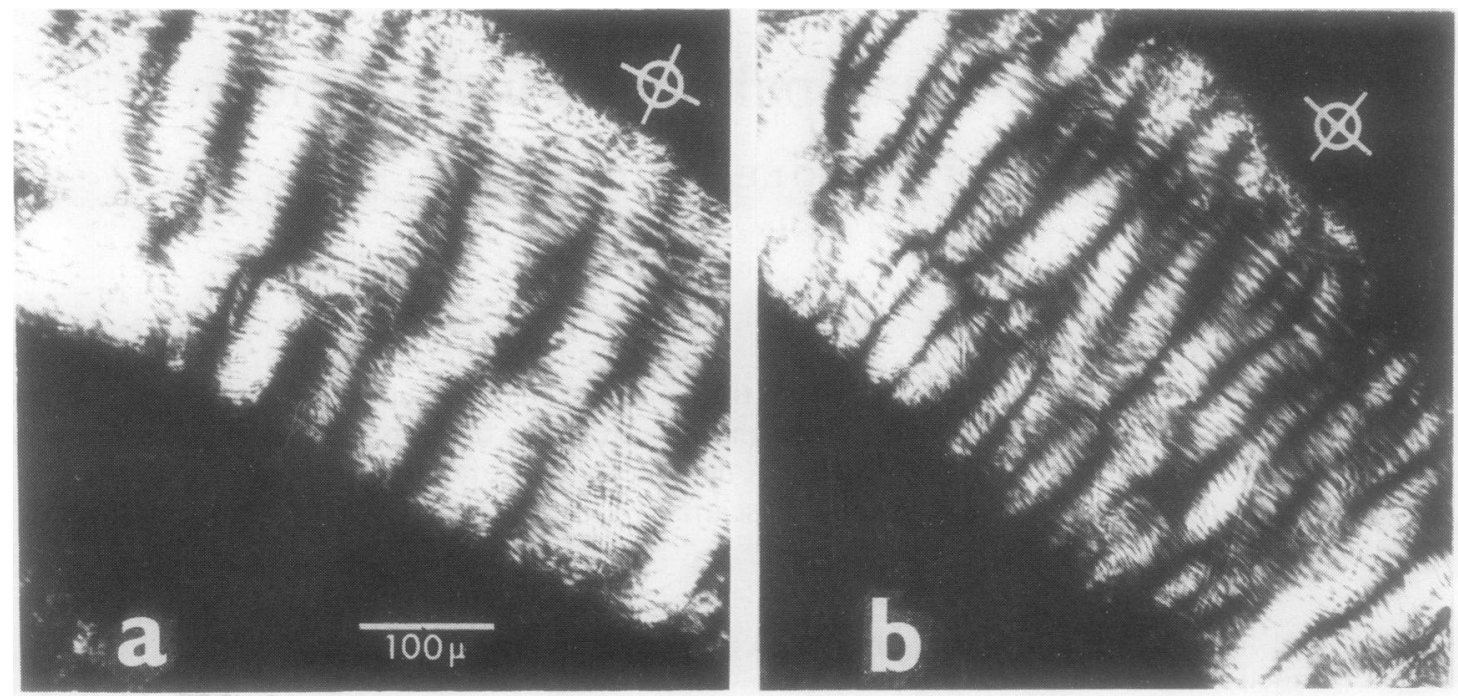

Fig. 2 Typical band patterns obtained on PM from palmaris longus tendon. (a) Angle of rotation $\theta=0^{\circ}$.

(b) Angle of rotation $\theta=11 \cdot 5^{\circ}$.

layer of peritenon fibres which normally masks the texture and morphology of the underlying fibres. From the above studies it was further speculated that the crimps are not present in the fibres of other human tissues.

In view of the findings of the survey of a large number of tissues, including human spinal ligaments, ${ }^{4-7}$ in which crimps are present, we report here a full and systematic reinvestigation of human palmaris longus tendon using PM and low angle $x$-ray diffraction. We also report SEM studies of this tissue using the recently developed new technique for direct revelation of the fibre morphology by SEM. ${ }^{16}$

\section{Materials and methods}

Palmaris longus tendons were obtained from postmortem material. Tendons for this study were selected from 4 subjects with no tendon related diseases and from an age range of 35-85 years. Immediately after removal the tendons were placed in physiological saline, buffered at $\mathrm{pH} 7 \cdot 4$, and frozen in liquid nitrogen. All specimens were subsequently stored at $-20^{\circ} \mathrm{C}$ until required. Sections of thickness about $0.5 \mathrm{~mm}$ were used for further analysis and were always taken from the outside of the tendon, where damage caused by ice-crystal formation during freezing would be minimal. Four specimens from each subject were examined by the following methods.

Polarising microscopy. For polarisation microscopy each specimen was mounted in a specimen holder designed by Shah et al. ${ }^{7}$ which permitted rota- tion of the specimen around its own axis. The specimen holder was then placed on the rotating stage of a polarising microscope. Under crossed polarising filters a pattern of light and dark bands along the fibre becomes visible. Following the procedures described by Diamant et al. ${ }^{3}$ and Shah et al. ${ }^{6}$ the variations of band patterns due to rotation around the microscope axis (angle of rotation designated $\theta$ ) and due to rotation around the specimen (fibre) axis (angle of rotation designated $\rho$ ) were documented. In line with previous workers orientation $\rho=0$ refers to the position in which the crimp plane of the fibres, if it exists, is perpendicular to the axis of the microscope. ${ }^{9}$

Following the recent work on RTT $^{16}$ fibre specimens were squashed between two microscope slides in two planes perpendicular to each other (locations of these planes corresponded to the orientation of each specimen designated by either $\rho=0^{\circ}$ or $\rho=$ $90^{\circ}$ ). The significance of these experiments is discussed later.

$S E M$. Recently a new method has been developed which enables easy removal of the outer layers of tendons without damaging the overall organisation of underlying layers. ${ }^{16}$ This method is based on the fact that tissues like skin and tendons swell in dilute solution of acetic acid, but the acid, without the aid of additional chelating agents, does not separate collagen from the interfibrillar matrix because even at low $\mathrm{pH} \approx 3$ some type of interaction still exists preventing dispersion of collagen fibrils. ${ }^{17}$ The method as applied to palmaris longus tendon is briefly described below. 
Fresh sections of the tendons were mounted in a stretching frame and held taught though not stretched. The whole assembly was immersed briefly (c. 30 seconds) in $1 \% \mathrm{v} / \mathrm{v}$ acetic acid (pH 3). This caused the outer sheath to swell. These swollen layers could then be removed by delicately running the edge of a supported needle along the specimen, the swollen and loosely held collagen readily adhering to the metal needle. Tendons were then dehydrated in $100 \%$ ethanol, critical point dried, and sputtercoated with gold before examination.

Low-angle $\mathrm{x}$-ray diffraction. The low-angle $x$-ray diffraction photographs of the tendons were taken by a Rigaku-Denki camera using $0 \cdot 2^{\circ}$ divergent (collimated) $\mathrm{CuK}_{\alpha} x$-ray beam and specimen to film distance of $200 \mathrm{~mm}$. Specimens were enclosed in saline moistened Lindemann tubes to prevent dehydration.

\section{Results and Discussion}

POLARISING MICROSCOPY

Fig. 2 illustrates the typical band patterns obtained from pieces of palmaris longus tendon. Changes in the band pattern on rotation around the microscope axis were found to be identical to those observed in other tendons and ligaments where crimps are known to be present. For example, Fig. 2a shows a band pattern for an angle of rotation $\boldsymbol{\theta}=0^{\circ}$ (so designated when the fibre axis coincides with the polarisation axis of one of the polarising filters). Fig. $2 b$ shows the band pattern when the specimen is rotated to $\theta \simeq 11^{\circ}$. In the first case the dark bands are narrow, in the second they have reached their broadest and are equivalent in width to the light bands.

The changes of the band patterns on rotation of the specimen around its own axis were also identical to those described in the crimped fibres of RTT. On the basis of the above evidence alone it could be concluded that fibres of palmaris longus tendons are crimped. The characteristic crimp parameters of sev- eral specimens, obtained from such changes in polarising patterns, are listed in Table 1 .

Figs. $3 a$ and $b$ and $4 a$ and $b$ illustrate the polarising patterns and interference contrast images of palmaris longus tendons squashed in the $\rho=0^{\circ}$ and $\rho=90^{\circ}$ positions. A squashed specimen in the $\rho=0^{\circ}$ position retains banding pattern in PM and therefore the crimps (Fig. 3a). The interference contrast image of the same specimen (Fig. 4a) also shows that the waveform of the fibres is maintained on squashing in the plane in which it is squashed (i.e., $\rho=0^{\circ}$ ). Tendon fibres compressed in the plane corresponding to $\rho=$ $90^{\circ}$ on the other hand do not show banding on PM (Fig. 3b). The interference contrast image (Fig. 4b) confirms that the fibres are no longer wavy.

The disappearance of the band pattern (and the waviness-Fig. 4b) in a specimen squashed in the plane $\rho=90^{\circ}$ cannot be explained if the origin of band pattern seen by PM of fresh and intact specimen is in the helical twisting of the fibres. This is so because the helical geometry would be symmetrical around the axis of the fibre (tendon), and squashing the specimen in any orientation would show nearly identical band pattern and waviness. On the other hand the above observations can be easily explained if it is assumed that the initial crimp waveforms were planar. Squashing the tendon in the crimp plane should keep the apices of the crimps intact. If, however, a tendon is squashed in such a way that the compression force is in the same direction as the 'amplitude' of the crimped wave form, then the crimps can be straightened out. Thus Figs. 3a, b, and $4 a, b$ together would indicate that the crimped fibres in the original unsquashed and intact fibres were crimped and arrayed in a planar way.

\section{S EM}

Figs. 5a, b are a stereo pair obtained by SEM of a section of palmaris longus tendon that has been stripped of its sheath. The stereoscopic view presented is

Table 1 Crimp parameters ( $\theta=$ crimp angle, $1=$ segment length) for human palmaris tendon of different ages. Columns 2 and 3 show the effects of alcohol dehydration and the acid stripping preparation on these parameters

\begin{tabular}{|c|c|c|c|c|}
\hline $\begin{array}{l}\text { Age } \\
\text { (years) }\end{array}$ & 0 & $\begin{array}{l}1 \\
\text { Normal }\end{array}$ & $\begin{array}{l}2 \\
\text { Alcohol } \\
\text { dehydrated }\end{array}$ & $\begin{array}{l}3 \\
\text { Acid treated and } \\
\text { alcohol dehydrated }\end{array}$ \\
\hline 35 & $\begin{array}{r}\theta^{\circ} \pm 1.96 \mathrm{se} \\
1(\mu) \pm 1.96 \mathrm{se}\end{array}$ & $\begin{array}{l}14 \cdot 8 \pm 1 \cdot 04 \\
24 \cdot 5 \pm 1\end{array}$ & $\begin{array}{l}16 \cdot 1 \pm 1 \cdot 2 \\
27 \cdot 2 \pm 2\end{array}$ & $\begin{array}{l}17 \cdot 4 \pm 1 \\
26 \cdot 7 \pm 1 \cdot 5\end{array}$ \\
\hline 63 & $\begin{array}{r}\theta^{\circ} \pm 1.96 \mathrm{se} \\
1(\mu) \pm 1.96 \mathrm{se}\end{array}$ & $\begin{array}{l}13 \cdot 3 \pm 1 \cdot 01 \\
20 \cdot 0 \pm 0.5\end{array}$ & $\begin{array}{l}15 \cdot 2 \pm 1 \cdot 12 \\
21 \cdot 2 \pm 0 \cdot 7\end{array}$ & $\begin{array}{l}15 \cdot 0 \pm 1 \cdot 02 \\
23 \cdot 4 \pm 1.9\end{array}$ \\
\hline 85 & $\begin{array}{r}\theta^{\circ} \pm 1.96 \mathrm{se} \\
l(\mu) \pm 1.96 \mathrm{se}\end{array}$ & $\begin{array}{l}11 \cdot 5 \pm 1 \cdot 1 \\
25 \cdot 7 \pm 1\end{array}$ & $\begin{array}{l}14 \cdot 2 \pm 2 \\
26 \cdot 2 \pm 0 \cdot 7\end{array}$ & $\begin{array}{l}15 \cdot 1 \pm 1 \\
27 \cdot 2 \pm 1 \cdot 1\end{array}$ \\
\hline 85 & $\begin{array}{r}\theta^{\circ} \pm 1.96 \mathrm{se} \\
l(\mu) \pm 1.96 \mathrm{se}\end{array}$ & $\begin{array}{l}10 \cdot 1 \pm 1 \cdot 96 \\
31 \cdot 8 \pm 4 \cdot 3\end{array}$ & $\begin{array}{l}13 \cdot 9 \pm 1 \cdot 56 \\
32 \cdot 0 \pm 3 \cdot 13\end{array}$ & $\begin{array}{l}14 \cdot 2 \pm 1 \cdot 31 \\
28 \cdot 75 \pm 4 \cdot 11\end{array}$ \\
\hline
\end{tabular}


480 Nicholls, Gathercole, Shah
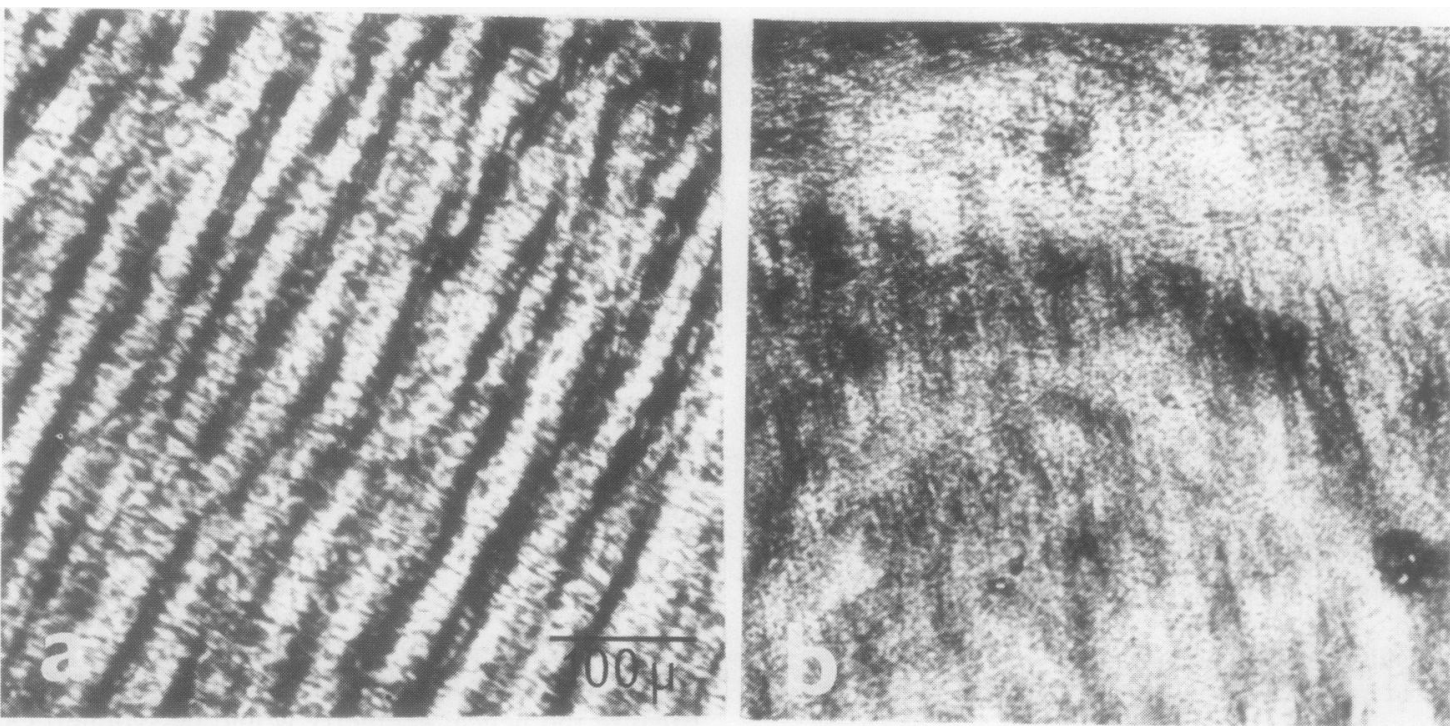

Fig. 3 Band pattern obtained in PM from palmaris longus squashed in (a) $\rho=0^{\circ}$, (b) plane $\rho=90^{\circ}$.
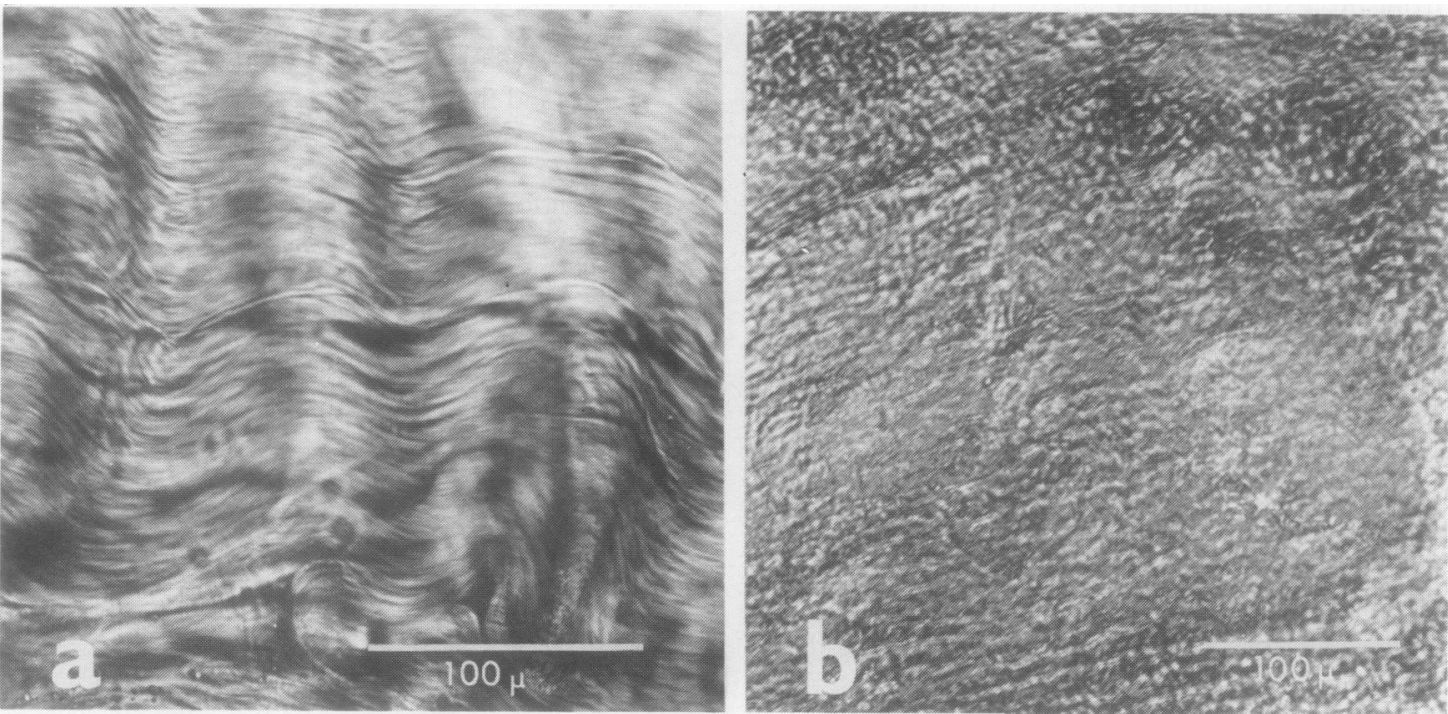

Fig. 4 Interference contrast image of the same specimens as in Fig. 3. Squashed in (a) plane $\rho=0^{\circ}$, (b) plane $\rho=90^{\circ}$.
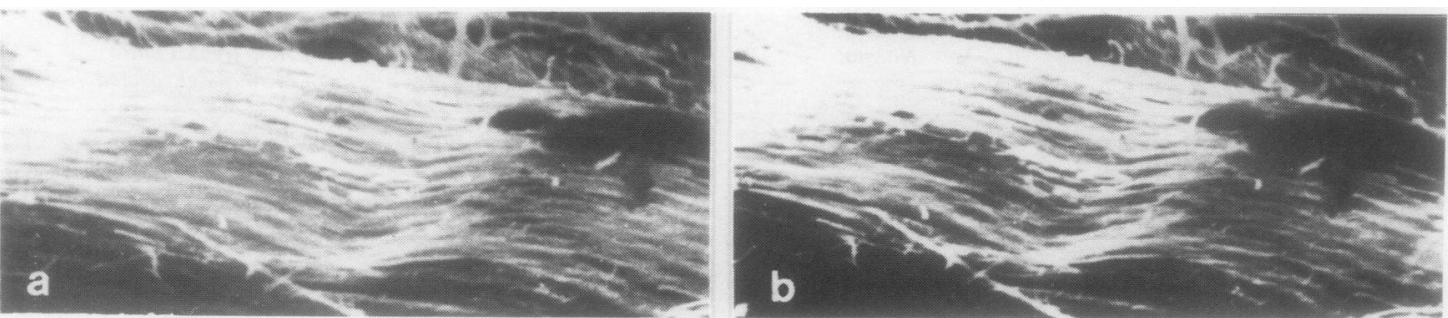

Fig. 5 Stereo pair scanning electron micrographs (a left, b right) of palmaris longus tendon with outer fibre layers removed. 
from such a direction that it confirms that it is correct to assign a crimp plane to the wavy nature of the fibres.

Fig. 6 shows a tendon section which is slightly more affected by the acid action. This clearly reveals intertwining of the individual subfibres, but the intertwining is at a much lower scale than the more prominent half wave periodicity- $25 \mu \mathrm{m}$, which corresponds to the periodicity of the banding pattern seen on PM.

The effects of preparative procedures in removing the outer layers for the revelation of the crimp-wave forms in SEM on the actual crimp parameters $(\theta, 1)$ have been quantified for RTT using measurements of $\theta$ and $l$ on PM by Nicholls et al. ${ }^{16}$ The changes are less marked in the case of human palmaris tendon (Table 1). The crimp parameters indeed change on dehydration by alcohol by $10 \%$, but the shape of the waveform itself does not change very much. Furthermore, there are no detectable differences between the crimp parameters of the tendons that have merely been dehydrated and those that have had prior processing in ace tic acid.

The artefacts of hyaluronidase treatment on the fibres of human palmaris longus tendon do not appear to have been elucidated systematically by Evans and Barbanel ${ }^{12}$ during the course of their investigation.

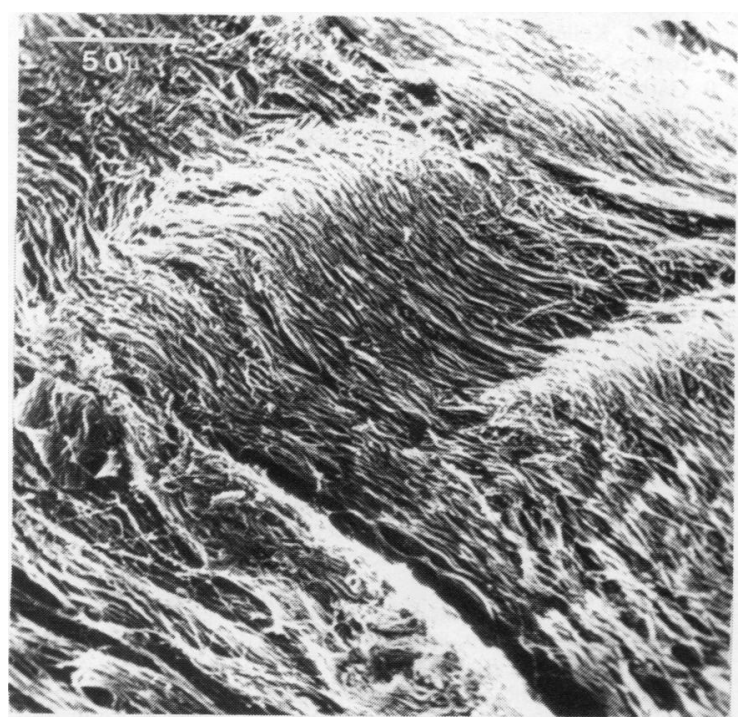

Fig. 6 Scanning electron micrograph of palmaris longus tendon, more deeply affected by the acid treatment and revealing intertwining nature of individual subfibres. Note that this intertwining is quite distinct from the main crimp waves with a periodicity of c. $25 \mu \mathrm{m}$.
LOW-ANGLE $x$-RAY DIFFRACTION

Figs. 7a and b show typical low-angle $x$-ray diffraction patterns obtained in $\rho=0^{\circ}$ and $\rho=90^{\circ}$ orientations. The patterns show reflections caused by the D-periodicity of collagen. In the native hydrated tendon this periodicity is $c .67 \mathrm{~nm}$ and is longer than that observed in fixed tendons, $c$. $64 \mathrm{~nm}$ (see Brodsky et $\left.a l .{ }^{18}\right)$. The patterns show essential features of planar crimping fully described previously. ${ }^{5}$ In the pattern for $\rho=0^{\circ}$ orientation, apart from the presence of 2 dominant sets of reflections-corresponding to the orientation of crimp arms-one sees a number of crossing and overlapping reflections in each set. These effects arise because (1) the diameter of the collimated $x$-ray beam of $c .300 \mu \mathrm{m}$ is much greater than the crimp length, and (2) fibrils in each crimp arm or successive parallel arms (see Fig. 8 and below) are not exactly aligned.

In the tendon under consideration the orientations of fibres change relatively abruptly and consecutively every $25 \mu \mathrm{m}$ along the tendon length. Thus as schematically shown in Fig. 8a, the $x$-ray beam incident on the tendon will sample several consecutive crimp arms 1, 2, 3, 4. Each crimp arm can be essentially regarded as individual diffractor with its own orientation. The centre of reflections $\left(\mathrm{C}_{1}, \mathrm{C}_{2}, \mathrm{C}_{3}, \mathrm{C}_{4}\right)$ pattern for each diffractor on the film are not coincident. (The recorded pattern contains symmetrically placed reflection streaks caused by $67 \mathrm{~nm}-\mathrm{D}$ periodicity). This is because the position of each diffractor with reference to the centre of the collimator is different (strictly speaking the plane of the film

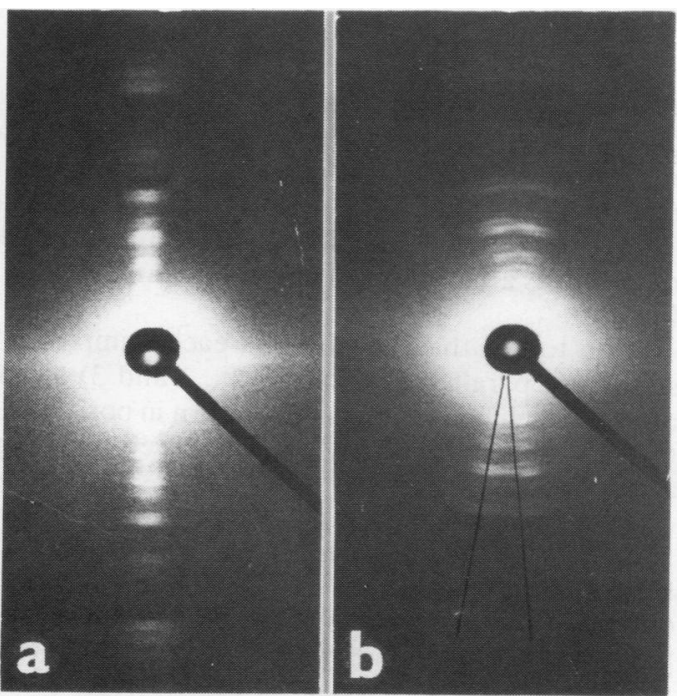

Fig. 7 Low angle $\mathrm{x}$-ray diffraction patterns of palmaris longus tendon obtained in orientations (a) $\rho=90^{\circ},(\mathrm{b}) \rho=0^{\circ}$. 


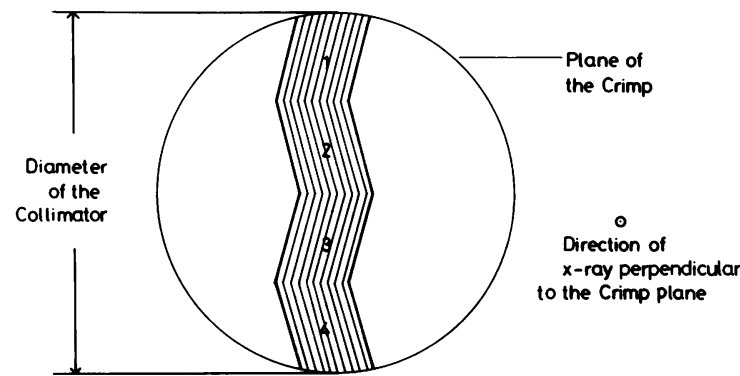

Fig. 8a Schematic representation of crimps enclosed by an $\mathrm{x}$-ray beam collimator. Crimps are orientated in the direction $p=0^{\circ}$ and therefore show the position of the specimen used for obtaining $\mathrm{x}$-ray patterns in Fig. 7a. Note that the $\mathrm{x}$-ray beam samples several consecutive crimp arms, e.g. 1, 2, 3, 4.

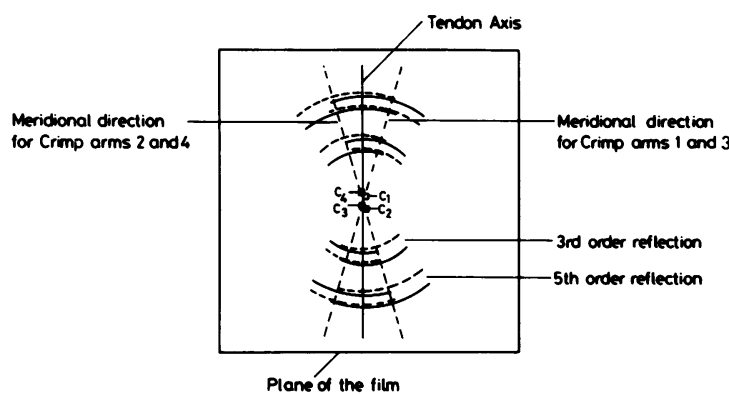

Fig. $8 \mathrm{~b}$ Schematic diagram of the low-angle $\mathrm{x}$-ray diffraction pattern from a specimen as positioned in Fig. 8 a $C_{1}, C_{2}, C_{3}, C_{4}$ are the centres of the pattern of diffraction lines (reflections) which are offset with respect to each other and the centre of the film. This offset causes relative radial shifts resulting in complex overlapping pattern.

intersects Evald's sphere slightly differently for each diffractor)-see Fig. 8b. This offset in the 'centres' of reflections manifests itself in relative radial shift in reflection, thus giving rise to multiplicity along the average meridional directions for 2 crimp arms (these radial shifts are even more predominantly evident in Fig. 7b).

If on top of this the fibrils in each crimp arm or successive parallel crimp arm (i.e., 1 and 3) are not absolutely aligned, then in the pattern in position $\rho=$ $0^{\circ}$ the radial shifts will be superimposed with slight azimuthal shifts. The net result of these effects is a complex pattern recorded in Fig. $7 \mathrm{~b}$.

In spite of the above complexities low-angle diffraction patterns form strong evidence that the crimped waviness in human palmaris tendon is also planar as it is in RTT.

The weight of the evidence derived from each and every technique utilised suggests that crimping with its planar waveform also exists in human palmaris tendon. No evidence whatsoever has been found in this investigation to indicate the presence of helically twisted fibres. We must therefore conclude that human palmaris tendon is not an exception to the empirical rule previously enunciated, namely: collagenous tissues which bear tension in vivo exhibit crimping.

The authors thank the ARC (S.P.N. and J.S.S.) and MRC (L.J.G.) for financial support, and Professor Andrew Keller for encouragement. They also acknowledge the help of Miss E. Hall, of the Royal United Hospital, Bath, in obtaining specimens.

\section{References}

1 Verzar F, Huber K. Die Strucktur der Sehnenfaser. Acta Anat (Basel) 1958; 33: 215-20.

2 Elliott D H. Structure and function of mammalian tendon. Biol Rev Cambridge Philosoph Soc 1965; 40: 392-421.

3 Diamant J, Keller A, Baer E, Litt M, Arridge R G C. Collagen ultrastructure and its relation to mechanical properties as a function of aging. Proc $R$ Soc Lond (Biol) 1972; 180: 293-315.

4 Dale W C, Baer E, Keller A, Kohn R R. On the ultrastructure of mammalian tendon. Experentia 1972; 28: 1293-5.

5 Gathercole L J, Keller A. Light microscopic wave forms in collageneous tissues and their structural implications. In: Atkins E D T, Keller A, eds. Structure of fibrous biopolymers. Colston Papers No. 26. London: Butterworth, 1975: 153-87.

6 Shah J S, Jayson M I V, Hampson W G J. Low tension studies of collagen fibres from the ligaments of the human spine. Ann Rheum Dis 1977; 36: 139-45.

7 Shah J S, Jayson M I V, Hampson W G J. Mechanical implications of crimping in collagen fibres of human spinal ligaments. Engineering Med 1979; 8: 95-102.

8 Torp S, Arridge R G C, Armeniades C D, Baer E. Structure property relationships in tendon as a function of age. In: Atkins E D T, Keller A, eds. Structure of fibrous biopolymers. Colston papers No. 26. London: Butterworth, 1975; 186-96.

9 Gathercole L J, Shah J S. Collageneous tissue morphology, a study of a substructure in rat tail tendons. In: Stokes I A F, ed. Mechanical factors and the skeleton. London: Libbey, 1981: 146-54.

10 Dlugosz J, Gathercole L J, Keller A. Transmission electron microscope studies and their relation to polarising optical microscopy in rat tail tendon. Micron 1978; 9: 71-82.

11 Kastelic J, Galeski A, Baer E. The multicomponent structure of tendon. Connect Tissue Res 1978; 6: 11-23.

12 Evans J H, Barbenel J C. Structure and properties of tendon related to function. Equine Vet $J$ 1975; 7: 1-8.

13 Gathercole L J, Keller A, Shah J S. Periodic wave pattern in native tendon collagen; correlation of polarising with scanning electron microscopy. J Microsc 1974; 102: 95-105.

14 Shah J S. (1977). Improvements in or relating to specimen stages for electron beam instruments. British Patent 1477458.

15 Shah J S, Beckett A. A preliminary evaluation of moist environment ambient temperature scanning electron microscopy. Micron 1979; 10: 13-23.

16 Nicholls S P, Gathercole L J, Keller A, Shah J S. Crimping in rat tail tendon collagen: morphology and transverse mechanical anisotropy. Int J Biol Macromol 1983; 5: 283-8.

17 Steven F S. The effect of chelating agents on collagen interfibrillar matrix interactions in connective tissue. Biochim Biophys Acta 1967; 140: 522-8.

18 Brodsky B, Eikenberry E F, Belbruno K C, Sterling K. Variations in collagen fibril structure in tendons. Biopolymers 1982; 21: $935-51$. 